\title{
Effect of stabilizer type on the physicochemical properties of poly(acrylic acid)/silver nanocomposites for biomedical applications
}

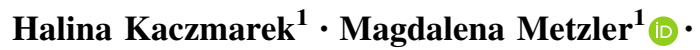 \\ Katarzyna Wegrzynowska-Drzymalska ${ }^{1}$
}

Received: 28 May 2015/Revised: 21 November 2015/Accepted: 2 February 2016/

Published online: 13 February 2016

(C) The Author(s) 2016. This article is published with open access at Springerlink.com

\begin{abstract}
Poly(acrylic acid), PAA, has been mixed in solution with silver nanoparticles, obtained by chemical reduction of silver nitrate. Two different sulfurcontaining organic compounds have been used as stabilizers of Ag nanoparticles: mercaptosuccinic acid (MSA) and 3-mercaptopropionic acid (MPA). The formation of Ag nanoparticles has been monitored by UV-Vis spectroscopy. The morphology and composition of obtained nanomaterials has been studied by electron microscopy techniques (SEM/EDX and HR-TEM). Nanomechanical properties of nanocomposites (adhesion and nanohardness) have been investigated by Atomic Force Microscopy (AFM). Thermal stability has been determined using thermogravimetric analysis. The exposure of specimens to UV radiation allowed to observe the changes in the nanoparticle structure and to estimate nanocomposite photostability. MSA has proved to be a better stabilizer of Ag nanoparticles immediately after the synthesis, when MPA provides better storage stability. MPA has allowed for the creation of nanoparticles using mixed reduction (chemical/photochemical) of $\mathrm{AgNO}_{3}$. It has been found that the nanocomposite materials are stable for 12 months of storage in solution and in solid form.
\end{abstract}

Keywords Poly(acrylic acid) - Nanosilver particles · Mercaptosuccinic acid · Mercaptopropionic acid · Nanocomposites characterization

Magdalena Metzler

wolnicka@doktorant.umk.pl

Halina Kaczmarek

halina@chem.umk.pl

Katarzyna Węgrzynowska-Drzymalska

kasiawd@doktorant.umk.pl

1 Faculty of Chemistry, Department of Chemistry and Photochemistry of Polymers, Nicolaus Copernicus University in Toruń, Gagarina 7, 87-100 Torun, Poland 


\section{Introduction}

Polymer nanocomposites containing noble metal nanoparticles are valuable materials for a wide variety of usage $[1,2]$. The possibility of their application in the production of biosensors [3, 4] catalysts [5] and ion-selective electrodes [6], surface-enhanced Raman scattering substrates [7] contributes to the high interest in these systems, also as materials for biomedical purposes [8]. The addition of silver nanoparticles to properly selected polymer matrix allows to give the whole nanocomposite biocidal properties. Bactericidal and fungicidal effect of silver-based nanomaterials has been repeatedly confirmed by scientific research.

The final properties of nanocomposites depend not only on the chemical structure and ratio of the components but also on the form of the modifier introduced (size and shape, and the degree of dispersion of the particles). Research efforts were focused on the preparation of nanoparticles with well-defined, uniform size and sufficiently high durability, which determines their practical use. The most popular methods of obtaining $\mathrm{Ag}$ nanoparticles are the methods of chemical and photochemical reduction of $\mathrm{AgNO}_{3}$ in the presence of the appropriate factors preventing agglomeration $[9,10]$. Size, shape and stability of nanoparticles can be planned by the selection of appropriate parameters such as initial silver salt and stabilizer concentration, molar ratio of the reducer and silver compound as well as the type of the applied stabilizer [11, 12].

Fine dispersion of nanosilver in a polymer can be obtained using two main approaches: (1) formation of colloidal particles in the presence of a polymer by reduction, and (2) dispersing of previously modified silver nanoparticles in a polymer [11].

Poly(acrylic acid) (PAA) and nanocomposites based on this polyelectrolyte can be considered as valuable materials in pharmaceuticals, cosmetics owing to its excellent properties such as water solubility, adsorption capability of different compounds, high hydrophilic nature, thickening and suspending properties. Thanks to the presence of carboxylic functional groups in each repeating unit, the PAA can be easily modified chemically. Due to the high water sorption, PAA and its derivatives are used in disposable diapers and sanitary materials, and because of the ability to efficient crosslinking it is used in the production of ions exchange resins, adhesives, paints and varnishes $[13,14]$. This polymer has been intensively studied by various experimental techniques, including AFM. For example, AFM tip was used to stretch PAA polymer chains and measure the deformation of a single chain under tension [15]. AFM is applied not only for imaging of surfaces; it supplies information on mechanical properties in nanoscale, particularly important in the case of application in nanotechnology. Furthermore, using the AFM adhesion force can be determined, which is meaningful in coating technology.

The aim of this work is to study the properties of PAA nanocomposites containing silver nanoparticles (AgNPs) stabilized by two different sulfur-containing compounds: mercaptosuccinic acid (MSA) and 3-mercaptopropionic acid (MPA). 
These stabilizers are selected because of their non-toxicity, thus they are used in the pharmaceutical industry [16-19]. MPA slightly different in chemical structure from MSA is known as an interesting compound having the ability to form a selfassembled monolayers (SAMs), which are applied in the nanocomposite field as molecular linkers. Moreover, MPA has been used for the preparation of biocompatible surfaces [16].

To characterize the properties of obtained PAA/AgNPs nanocomposites, including their nanomechanical properties, thermal and photochemical stability, several complementary instrumental methods (UV-Vis spectroscopy, scanning microscopy: SEM, HR-TEM, AFM, thermogravimetry) were used. The effect of the type of stabilizer on the tested properties has been compared.

\section{Materials and methods}

\section{Materials}

Monomer-acrylic acid, $\mathrm{CH}_{2}=\mathrm{CHCOOH}, \mathrm{AA}$ - was purchased from Sigma Aldrich (Saint-Quentin Fallavier, France). Mercaptosuccinic acid, $\mathrm{HOOC}-\mathrm{CH}(\mathrm{SH})-\mathrm{CH}_{2}$ $\mathrm{COOH}$, MSA; 3-mercaptopropionic acid, $\mathrm{CH}_{2}(\mathrm{SH})-\mathrm{CH}_{2} \mathrm{COOH}$, MPA and other reagents (sodium borohydride and silver nitrate) were supplied by Sigma Aldrich (Saint Louis, MO). Solvents - methanol and ethanol (anhydrous)—were purchased from POCh (nowadays-Avantor Performance Materials Poland S.A.). Before polymerization, the AA was distilled under reduced pressure for purification. The other reagents were of high-grade purity and were used without further purification.

\section{Synthesis of poly(acrylic acid)}

The purified monomer (acrylic acid, AA) was polymerized in aqueous solution in the presence of hydrogen peroxide as an initiator. The details of poly(acrylic acid) synthesis were described in the previous work [19].

\section{Synthesis of stabilized silver nanoparticles: Ag/MSA and Ag/MPA}

The preparation of Ag/MPA and Ag/MSA was based on the chemical reduction of $\mathrm{AgNO}_{3}$ by $\mathrm{NaBH}_{4}$ in the presence of 3-mercaptopropionic acid (Fig. 1a) or
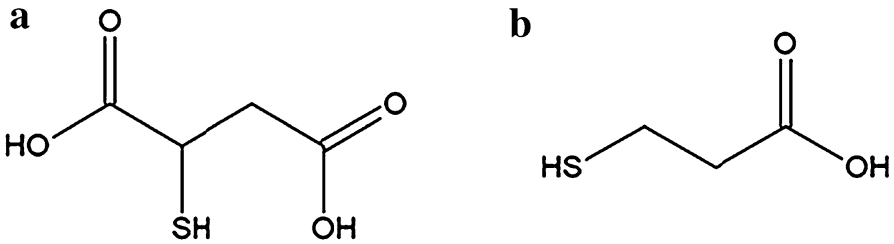

Fig. 1 The chemical structuranother thermal parameters,e of Ag nanoparticle stabilizers: MSA (a) and MPA (b) 
mercaptosuccinic acid (Fig. 1b) as a stabilizer. $\mathrm{AgNO}_{3}$ was dissolved in methanol and then added dropwise to the solution of stabilizers. The mixture was stirred at room temperature. Thereafter, $\mathrm{NaBH}_{4}$ was added to the solution and mixed. The obtained solution was cooled in the refrigerator for $3 \mathrm{~h}$. The products were filtered and washed by methanol. Finally, nanoparticles were dried at room temperature.

\section{Preparation of PAA/Ag/MPA and PAA/Ag/MSA nanocomposites}

Aqueous solution of Ag/MPA/NPs and Ag/MSA/NPs were mixed with solution of PAA and intensively stirred at room temperature overnight to obtain a homogeneous solution. The content of AgNPs introduced into a nanocomposite was $3 \%$ wt with respect to the weight of the polymer. Nanocomposites in the form of thin solid films have been obtained by casting of mixed solution on leveled polystyrene plates. After solvent evaporation, the samples were carefully dried in a dark place in vacuum at room temperature and gently removed from the support surface.

\section{Conditions of UV exposure}

The samples were exposed to high-pressure mercury vapor lamp (HPK $125 \mathrm{~W}$, Philips, Holland) in solution or in solid state in air atmosphere at room temperature. The lamp emitted polychromatic radiation $(\lambda=248-578 \mathrm{~nm})$. The intensity of incident light at the sample level, measured by electronic radiometer HD 9021 (Delta OHM, Italy) equipped with three probes for UVA, UVB and UVC ranges was $43.0 \mathrm{~W} / \mathrm{m}^{2}(315-400 \mathrm{~nm}), 35.25 \mathrm{~W} / \mathrm{m}^{2}(280-315 \mathrm{~nm})$ and $8.38(200-280 \mathrm{~nm})$ $\mathrm{W} / \mathrm{m}^{2}$, respectively.

\section{Analysis}

UV-Vis spectra of samples in solutions were obtained by means of UV-PC1600 Shimadzu spectrophotometer.

The TG, DTG and DTA curves were recorded using TA Instrument SDT 2920 Simultaneous DSC-TGA at temperature range of $20-600{ }^{\circ} \mathrm{C}$ and heating rate of $10{ }^{\circ} \mathrm{C} / \mathrm{min}$. The carrier gas was nitrogen at a flow rate of $100 \mathrm{ml} / \mathrm{min}$.

The SEM images were obtained by a microscope LEO Electron Microscopy Ltd, England and Quanta 3D FEG. An energy-dispersive X-ray (EDX) microanalysis was performed with a spectrometer Quantax 200 with XFlash 4010 (Bruker AXS) coupled to the scanning electron microscope.

The images of the high-resolution transmission electron microscopy (HR-TEM) were recorded with an FEI Europe; model Tecnai F20 X-Twin (2011) at high vacuum. The specimen for TEM was prepared by deposition of solution drop onto a copper grid.

Atomic force microscopy studies were performed at ambient conditions using the NanoScope IIIa Multimode microscope (Veeco, Santa Barbara, USA) with NanoScope IIIa controller. Indentation test with applying of silicon nitride tip (NP-1 with spring constant of $0.78 \mathrm{~N} \mathrm{~m}^{-1}$ ) has been performed for estimation of materials nanohardness. Dents were made at a constant force and rate, using the 
deflection of cantilever as a measure of the force. The three-sided pyramid diamond cantilever with $60^{\circ}$ apex angle (with spring constant of $859 \mathrm{~N} / \mathrm{m}$ ) has been used for adhesion measurements. The details of such analysis using AFM were described earlier [20, 21]. The presented results are the average of 10-40 measurements.

X-ray diffraction (XRD) experiments were carried out with an X'PERT Pro Philips Diffractometer $\left(\mathrm{Cu} K_{\alpha} 1\right.$, wavelength $1.54056 \AA$ A, range $2 \theta 5^{\circ}-120^{\circ}$, scan step size $0.020^{\circ}$, time per step $3.00 \mathrm{~s}$ ).

\section{Results and discussion}

\section{$\mathrm{UV}-\mathrm{Vis}$ spectroscopy}

Silver nanoparticles: generation and stability

The formation of silver nanoparticles during the reduction of $\mathrm{AgNO}_{3}$ was monitored by UV-Vis spectroscopy in aqueous solutions (Fig. 2). Nanoparticles were colored: powders were brown, while NPs solutions were brownish (Ag/MSA) or yellowish (Ag/MPA), thus absorption band in the visible range appeared. This band can be assigned to surface plasmon resonance caused by the collective oscillation of conducting electrons typical for nanoparticles of noble metals [22]. Generally, the shape and width of absorption band depend on the particles size distribution [9]. The bands located at shorter wavelengths are assigned to smaller nanoparticles than these at longer wavelength range. Moreover, when the absorption peak is sharp and symmetric, the nanoparticles are characterized by a low size distribution. In our case the plasmon bands are relatively broad, which indicates the polydisperse of NPs.

In the case of using MPA as a stabilizer, an initial plasmonic band (at $\sim 350 \mathrm{~nm}$ ) is characterized by a very low intensity (Fig. 3a). The high turbidity (observed as

Fig. 2 UV-Vis absorption spectra of Ag/MPA, PAA/Ag/ MPA, Ag/MSA and PAA/Ag/ MSA directly after preparation

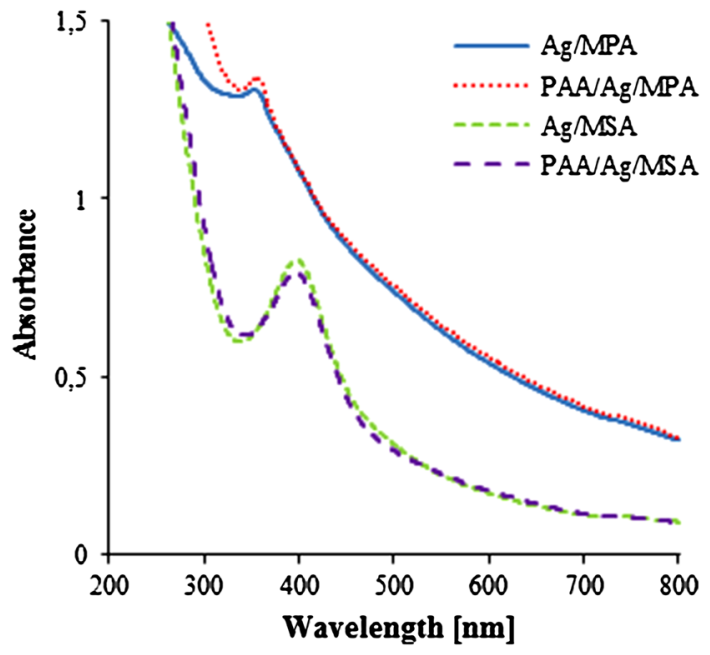



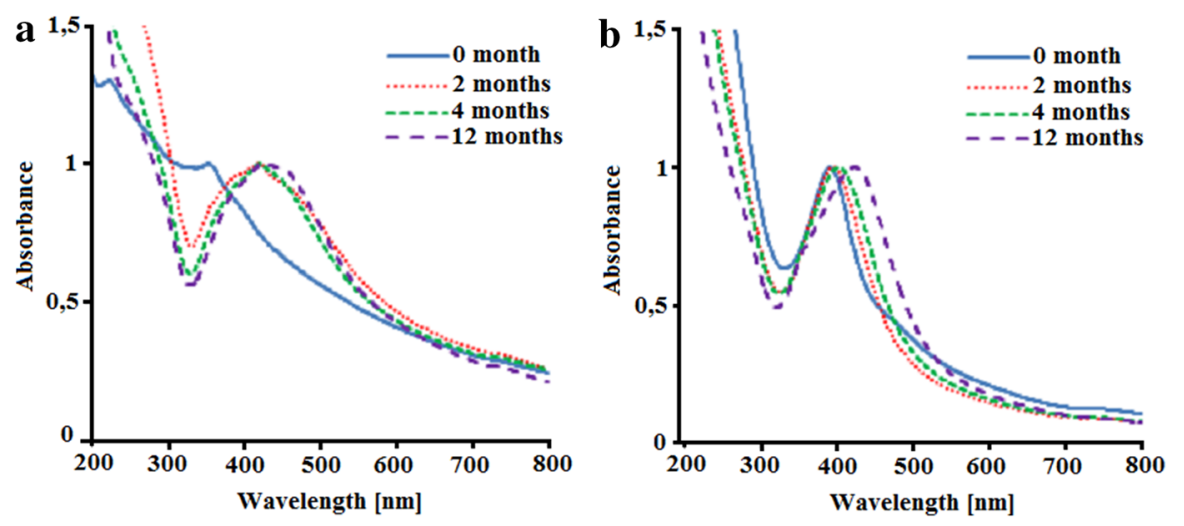

Fig. 3 Normalized electron absorption spectra of Ag/MSA (a) and Ag/MPA (b) after long-term storage in dark

high background at UV-Vis spectrum) is caused by scattering of incident light due to the presence of large colloidal particles. However, storage of solution in the dark for 2 months resulted in a distinct increase of absorption band with a maximum at $418 \mathrm{~nm}$, which means that the reaction in the system continues. This is related to the kinetics of formation of nanoparticles, as described by He et al. [9]. In this work too, directly after completion of the addition of the reducing agent $\left(\mathrm{NaBH}_{4}\right)$ to $\mathrm{AgNO}_{3}$ solution, there was no clear absorption.

Further storage of our Ag/MPA solution for 2-12 months does not cause any changes in the position of maximum absorption band (at about $420 \mathrm{~nm}$ ). In addition, the intensity of the analyzed band varies slightly at this time.

As can be seen from Fig. 3b, Ag nanoparticles stabilized by MSA are formed directly after reduction of silver salt and they are relatively stable during prolonged storage. In UV-Vis spectrum of Ag/MSA solution left in the dark for 12 months, absorption maximum was shift towards longer wavelengths (from 395 to $425 \mathrm{~nm}$ ), moreover, band broadened, indicating an increase in nanoparticle size [23]. It means that Ag nanoparticles stabilized by MSA undergo slow agglomeration. Similar plasmon absorption pattern has been reported previously for Ag/MSA by Sumesh et al. [2]. The position of maximum was independent on the component ratio ( $\mathrm{Ag} /$ MSA) but the bandwidth was narrower in the case of higher stabilizer content.

As expected, both types of solid silver nanoparticles (Ag/MPA and Ag/MSA) do not change during 1-year storage at room temperature in dark. This is the evidence of effective stabilizing effect of MSA and MPA by formation of permanent bonding of thiol groups with a silver. Such bonds also efficiently stabilize the dispersion of metal nanoparticles in organic systems [11].

It is a valuable advantage of obtained nanocomposite, as opposed to particles that may precipitate from colloidal system losing their properties within 1 week, as for example, these are described in the article on the influence of the type of precursor, reducing agent and stabilizer on the shape and size of type of NPs [12]. 


\section{PAA/AgNPs nanocomposites}

The pure components used for preparation of nanocomposites (i.e., PAA, MSA, MPA) do not contain chromophoric groups absorbing in the visible light. Only the introduction of the $\mathrm{Ag}$ nanoparticles into polymer solution produces color and causes the plasmon absorption.

PAA/Ag/MPA and PAA/Ag/MSA nanocomposites exhibit similar spectral behavior to stabilized Ag NPs in aqueous solutions. In the first-mentioned system, the band appears at about $355 \mathrm{~nm}$, while in the spectrum of the second nanocomposite at $395 \mathrm{~nm}$. The obtained spectra are similar to presented in the literature also for Ag nanoparticles stabilized by other polymers such as poly(vinyl pyrrolidone) and poly(vinyl alcohol) [12].

However, there was a difference in the case of PAA/Ag/MSA and Ag/MSA. The absorption maximum shift in spectra does not occur in the presence of the polymer during storage, as it was in the system of alone Ag/MSA nanoparticles. This indicates the additional stabilizing effect of the PAA, which prevents agglomeration during aging of the stored material.

In PAA/Ag/MPA very weak, blurry band at $355 \mathrm{~nm}$ appears just after nanocomposite preparation, analogically as in Ag/MPA NPs. Also in this case, the changes during storage are negligible.

\section{Effect of UV irradiation on AgNPs and PAA/AgNPs nanocomposites}

Initially, UV irradiation (to about $15 \mathrm{~min}$ ) causes an increase of plasmon absorption band in both types of AgNPs. Similar effect in the early stages of irradiation (up to $24 \mathrm{~min}$ ) is observed in the PAA/Ag NPs nanocomposites. The changes in the absorption spectra are shown in the example of Ag/MPA and PAA/Ag/MPA (Fig. 4). In these samples, the increase in the intensity of the band is extremely large, reflecting the greater efficiency of photochemical processes in the formation of Ag nanoparticles compared to a chemical reaction. After 24-min exposure clear absorption band in the range of 330-500 $\mathrm{nm}$ with maximum absorption at $416 \mathrm{~nm}$ is observed in Ag/PMA and PAA/Ag/MPA spectra. It has been found in literature that also high-energy radiation has the significant effect on formation of AgNPs: when
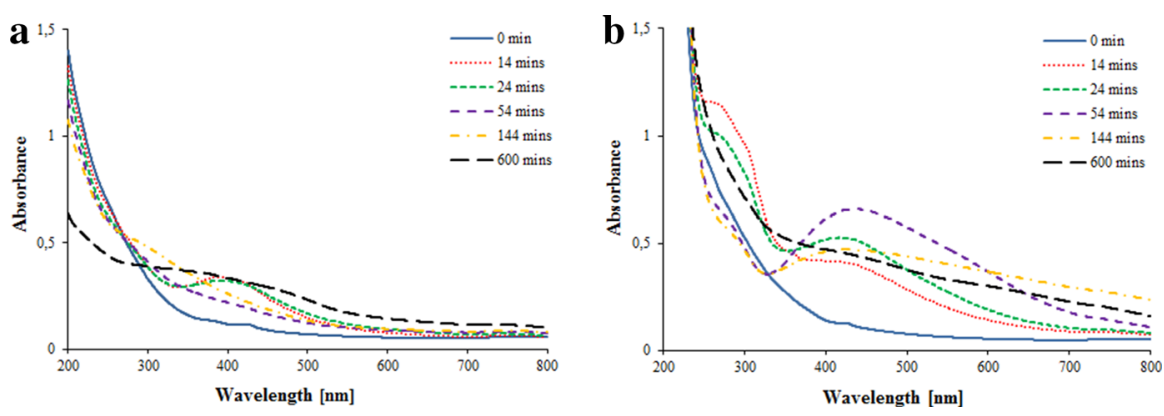

Fig. 4 Changes of UV-Vis spectra of Ag/MPA (a) and PAA/Ag/MPA (b) during exposure to UV 


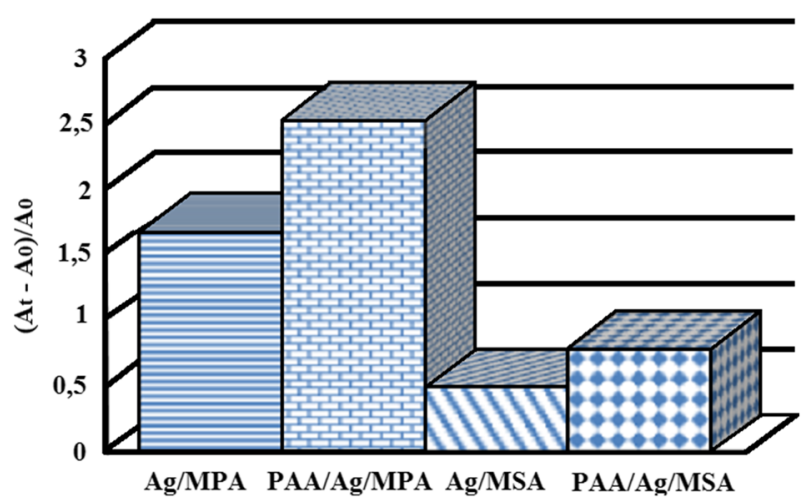

Fig. 5 The relative change in absorbance at maximum band for samples tested: Ag/MPA (a) and PAA/ Ag/MPA (b), Ag/SPA (c) and PAA/Ag/MSA (d)

the bigger Ag microparticles were bombarded by the electron beam, the small monodisperse nanoparticles with uniform shape were formed [10].

The longer action of UV irradiation (>50 min) influences $\mathrm{Ag}$ nanoparticles adversely causing the decrease of absorbance, which may indicate the material photodegradation. Mainly organic ingredients of the systems, i.e., stabilizers (MPA and MSA) and PAA matrix, should be susceptible to photochemical reactions. However, the spectra of the thiol acids treated by UV under the same conditions as the samples with nanoparticles changed insignificantly. This demonstrates the photochemical resistance of MPS and MSA in contrast to the PAA forming matrix. PAA photodegradation has been previously described [14, 24].

Comparison of photochemical stability systems investigated, estimated on the basis of spectroscopic studies, is shown in Fig. 5, where the relative changes of absorbance at band maximum after $600 \mathrm{~min}$ of UV irradiation are plotted. Although the maximum of band for different samples occur at somewhat different values $(349.5,355,395$ and $395 \mathrm{~nm}$ in spectrum of Ag/MPA, PAA/Ag/ MPA, Ag/MSA and PAA/Ag/MSA, respectively), this figure gives an idea on the behavior of AgNP-containing systems upon UV. Ag/MSA appeared more photostable than Ag/MPA but in PAA nanocomposites photostability decreases. However, these changes are very low in PAA/Ag/MSA comparing to PAA/Ag/ MPA.

\section{Thermogravimetric analysis (TGA)}

Figures 6 and 7 show thermogravimetric curves of the Ag/MPA, Ag/MSA, PAA/Ag/MPA and PAA/Ag/MSA specimens before and after $25 \mathrm{~h}$ UV irradiation. The thermal parameters obtained from thermal analysis are collected in Table 1. 


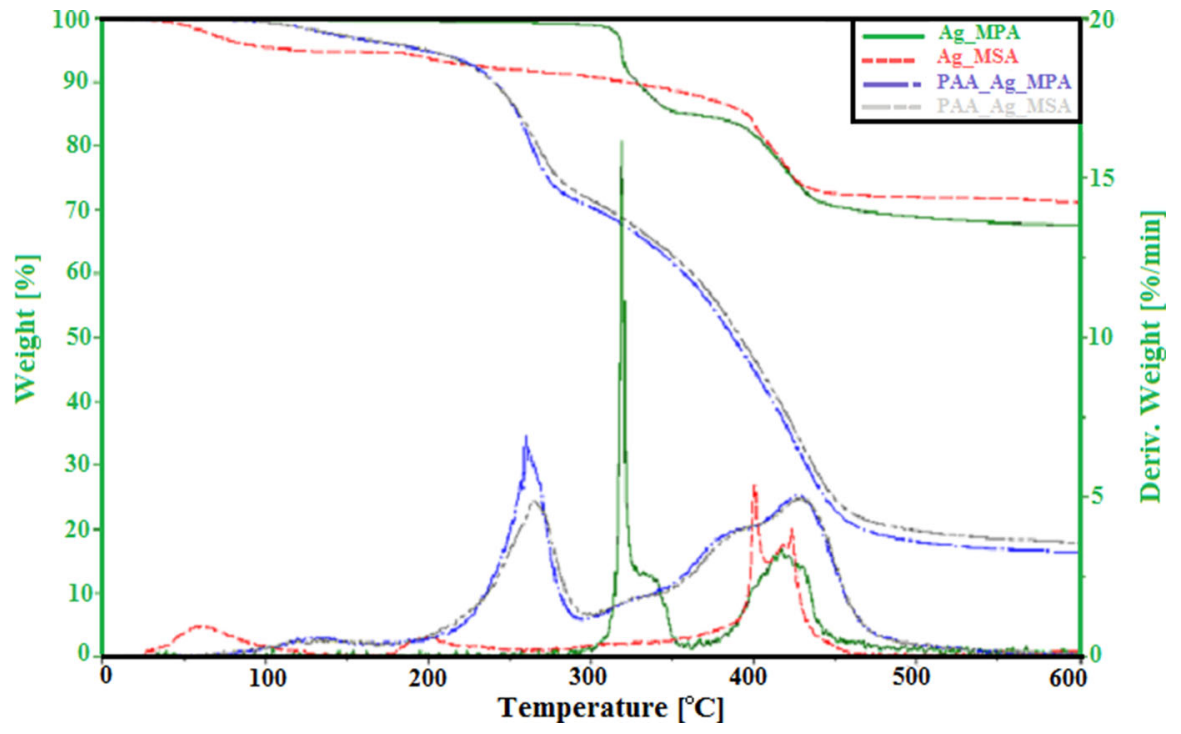

Fig. 6 TG and DTG curves of unirradiated Ag/MPA, Ag/MSA, PAA/Ag/MPA and PAA/Ag/MSA

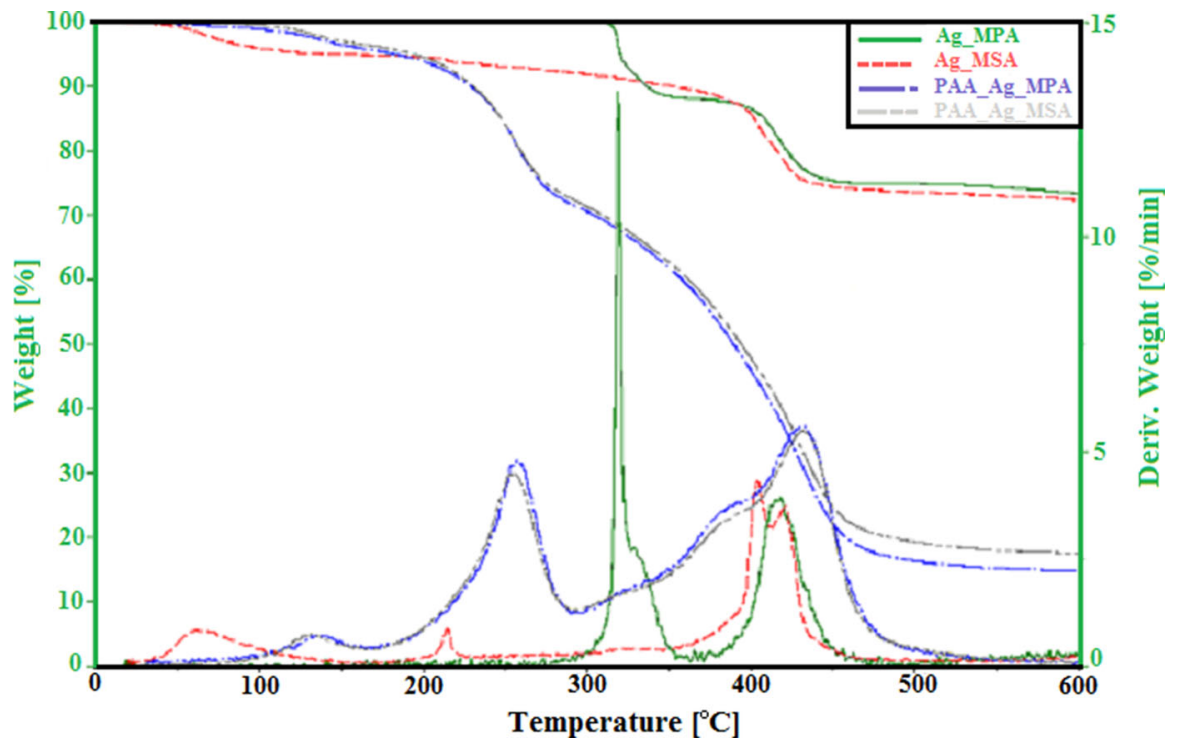

Fig. 7 TG and DTG curves of 25-h irradiated Ag/MPA, Ag/MSA, PAA/Ag/MPA and PAA/Ag/MSA

The initial small weight loss below $170{ }^{\circ} \mathrm{C}(4-5 \%)$, observed in all samples (except of Ag/MPA), can be attributed to the presence of moisture, difficult to remove during standard drying process. 


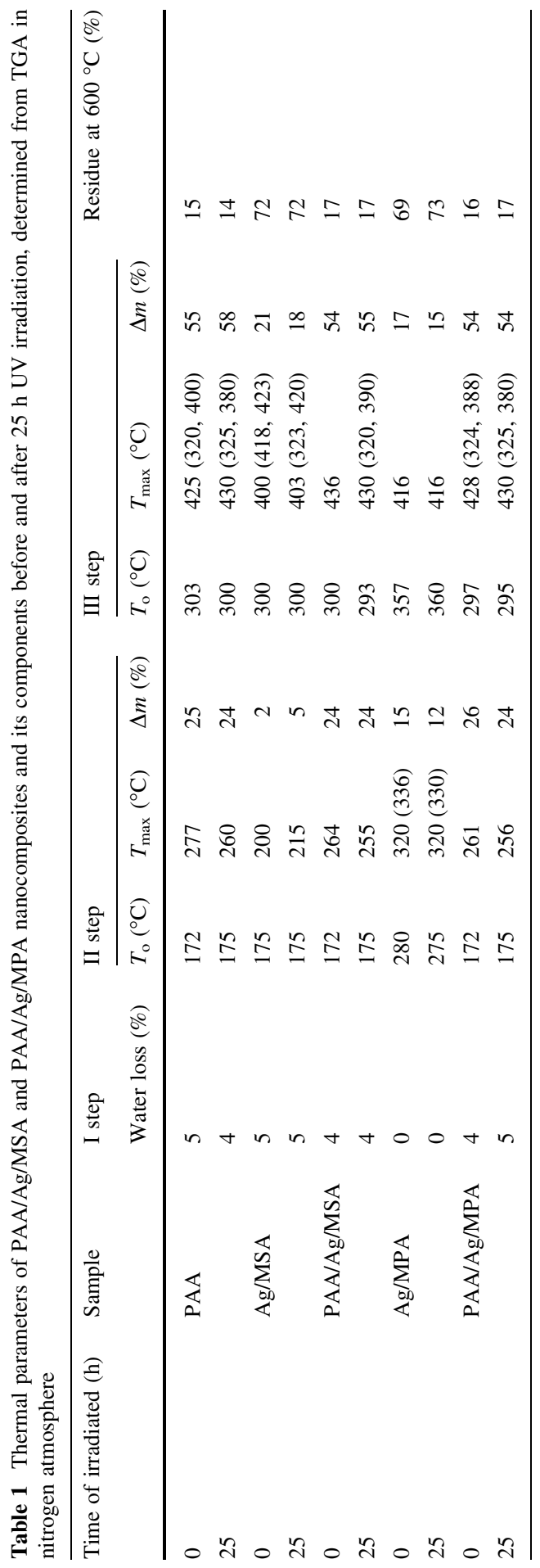




\section{Unirradiated samples}

PAA/Ag/MPA and PAA/Ag/MSA nanocomposite, heated to $600{ }^{\circ} \mathrm{C}$, decompose in three main steps (Fig. 6). Since the first step (in range of $50-150{ }^{\circ} \mathrm{C}$ ) is not associated with chemical changes of samples, we will focus on the discussion of the second main stage of thermal decomposition, starting above $200{ }^{\circ} \mathrm{C}$.

At $200-300{ }^{\circ} \mathrm{C}$ range occurs fast degradation with maximum rate at $261{ }^{\circ} \mathrm{C}$ for PAA/Ag/MPA and $264{ }^{\circ} \mathrm{C}$ for PAA/Ag/MSA (while in the case of pure PAA it was at $277^{\circ} \mathrm{C}$ ). In this step, the low molecular weight degradation products are emitted as a result of rupture of covalent bonds $[25,26]$. The corresponding weight loss is about $24-26 \%$.

The third, complex step of the nanocomposite degradation occurring at $300-500{ }^{\circ} \mathrm{C}$ range (with maximum rate at 436 and $428{ }^{\circ} \mathrm{C}$ in PAA/Ag/MSA and PAA/Ag/MPA, respectively) is related to the main chain scission and the evolution of other degradation products. The weight loss at this step is $54 \%$ for both nanocomposites (approximately the same as in the case of PAA alone).

Thermal decomposition of Ag NPs may be considered as a two-step process. Taking into account $T_{\max }$ value of the decay at first step, it can be concluded that Ag/MPA is a more stable system than Ag/MSA. Furthermore, Ag/MPA has not permanently bound water, which can affect its properties. Probably in Ag/MPA, the formation of hydrogen bonds between the MPA particles is favored, whereas in the $\mathrm{Ag} / \mathrm{MSA}$, water and MSA molecules interact forming durable hydrogen bonds.

The content of Ag calculated on the base of residue at $600{ }^{\circ} \mathrm{C}$ is about $70 \%$ in both cases (Ag/MPA and Ag/MSA). Thus, the content of pure Ag NPs in PAA nanocomposite can be estimated at approximately $2 \%$.

Summing up this part of the study, looking at the onset of the decomposition $\left(T_{\mathrm{o}}\right.$ of first stage) it can be concluded that there is no significant difference in thermal stability of nanocomposites and virgin PAA. However, taking into account $T_{\max }$, the observed decrease of about a dozen degrees in the presence of AgNPs can indicate the certain acceleration of PAA thermal degradation in unexposed and exposed samples. It suggests the catalyzing action of silver analogically as in the case of thermal degradation of PAA in the presence of copper compounds [25].

\section{$U V$-irradiated samples}

The same specimens as in previous section have been UV irradiated up to $25 \mathrm{~h}$ and again subjected to the thermal analysis (Fig. 7). As presented in Table 1, the content of the physically adsorbed water and carbon residue at $600{ }^{\circ} \mathrm{C}$ is almost the same in the exposed PAA, PAA/Ag/MPA and PAA/Ag/MSA samples. The main difference is observed in the second step- $T_{\max }$ in the UV-irradiated PAA, PAA/AgMPA and PAA/Ag/MSA decreases (comparing to unirradiated samples). In addition, $T_{\mathrm{o}}$ of the third decomposition step is lower by about 2-6 degrees than the corresponding values for the unexposed samples. It can be explained by formation of thermally unstable products during the UV exposure. 
However, another thermal parameters, including the weight loss and carbon residue at the end of heating $\left(600{ }^{\circ} \mathrm{C}\right)$ in PAA, PAA/Ag/MPA, PAA/Ag/MSA and $\mathrm{Ag} / \mathrm{MSA}$ are almost the same before and after the UV action.

\section{Scanning electron microscopy (SEM) and energy-dispersive X-ray spectroscopy (EDX)}

SEM allows to observe the surface morphology of PAA/Ag nanocomposites. It was found that silver nanoparticles are evenly scattered in the poly(acrylic acid) matrix.

Examples of SEM images of Ag/MPA and Ag/MSA nanoparticles embedded in PAA are shown in Fig. 8. These images show agglomerates of different shapes: Ag/ MPA NPs create oval structure or less regular clusters while Ag/MSA are arranged in the form of a ribbon, more or less tangled.

The estimated size of the aggregates in each case is in the range of 100-400 nm. The diameter of the individual nanoparticles present in agglomerates can be estimated at approximately $10-15 \mathrm{~nm}$.

The elemental composition of PAA/Ag/MPA and PAA/Ag/MSA was investigated by EDX analysis. In particular, the content of silver and other present elements (carbon, oxygen and sulfur) at the surface of PAA/Ag/MPA and PAA/Ag/ MSA films is given in Table 2. The weight fraction of Ag depends on the location of the area selected for this study. Because of the great variation in the composition at different measuring points, it makes no sense to count average values.

The data presented in the first row of the Table 2 show the percentage composition of elements obtained for the entire scan area. Values for PAA/Ag/MPA in lines 2, 3, 4 as well as for PAA/Ag/MSA in lines 1, 2, 4 respond to areas, in which the silver presence was expected (bright points in the SEM/EDX picture). Moreover, the analysis of the darker areas showed that silver nanoparticles appear inside the matrix, which is confirmed by the results of the percentage composition of silver for PAA/Ag/MPA and PAA/Ag/MSA which is 0.70 and 0.08 , respectively.
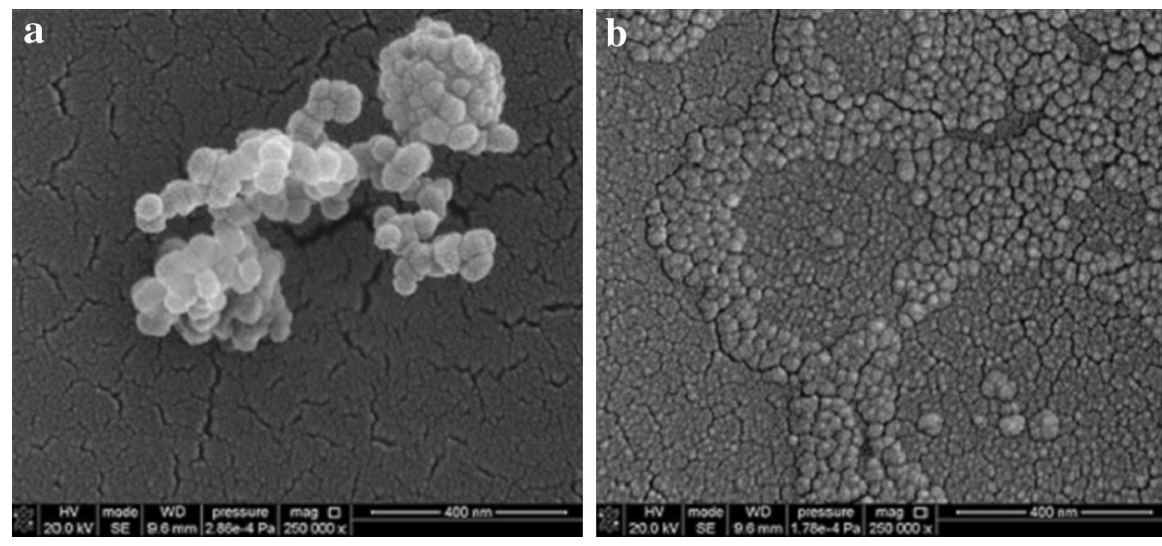

Fig. 8 SEM image of PAA/Ag/MPA (a) and PAA/Ag/MSA (b) films 
Table 2 Content of elements (\%wt) in studied nanocomposites obtained from EDX/SEM analysis

${ }^{\text {a }}$ Data obtained for the whole scan area

\begin{tabular}{lllll}
\hline Sample & \multicolumn{4}{l}{ Element $(\mathrm{wt} \%)$} \\
\cline { 2 - 5 } & $\mathrm{C}$ & $\mathrm{O}$ & $\mathrm{S}$ & $\mathrm{Ag}$ \\
\hline PAA/Ag/MPA & 23.12 & 74.82 & 0.49 & $1.57^{\mathrm{a}}$ \\
& 24.56 & 71.19 & 1.14 & 3.12 \\
& 24.14 & 68.99 & 1.69 & 5.19 \\
& 23.22 & 70.61 & 1.49 & 4.68 \\
& 28.57 & 70.50 & 0.22 & 0.70 \\
PAA/Ag/MSA & 17.25 & 73.43 & 3.15 & 6.16 \\
& 36.81 & 62.07 & 0.11 & 0.77 \\
& 36.70 & 62.98 & 0.06 & 0.08 \\
& 32.93 & 66.1 & 0.14 & 0.64 \\
\hline
\end{tabular}

Although the method provides quantitative information about the composition of elements, in this case, the heterogeneity of nanocomposite is responsible for data scatter.

It should be noted that the SEM/EDX analysis involves testing the sample to a depth of about $2-5 \mu \mathrm{m}$. In the case of studied specimens, the silver nanoparticles obviously appear not only at the surface, but also in deeper polymer layers. The accuracy of EDX spectrum is affected by numerous factors (e.g., sample density, inhomogeneity and roughness, overlapping peaks of different elements), therefore, distribution of the silver in different sample points is relatively high.

It can be concluded that in the studied systems, Ag NPs are present on the surface of nanocomposites, which is very important from the point of view of the biocidal action of these systems during practical applications.

\section{High-resolution transmission electron microscopy (HR-TEM)}

Observations by HR-TEM provide additional information about the internal structure of nanocomposites. The single nanoparticles of both types (Ag/MSA and Ag/MPA) take in a matrix of PAA very regular spherical shapes (Fig. 9a). The size of nanoparticles observed on the HR-TEM images $(10-80 \mathrm{~nm})$ are consistent with data obtained by SEM method. Enlargement of single nanoparticle allows the visualization of the regular structure of silver NPs (Fig. 9b).

The calculated $d$ spacing equals 0.2343 and $0.2390 \mathrm{~nm}$ for Ag/MPA and Ag/ MSA, respectively. This corresponds to the literature values for the metallic silver [27].

\section{X-ray diffraction analysis of PAA/Ag nanocomposites}

Presence of metallic silver in PAA nanocomposites has been confirmed by XRD. An example of X-ray diffraction pattern of PAA/Ag/MSA is shown in Fig. 10. The characteristic signals at $2 \Theta: 38^{\circ}, 44^{\circ}, 64^{\circ}$ and $77^{\circ}$, corresponding to (111), (200), 

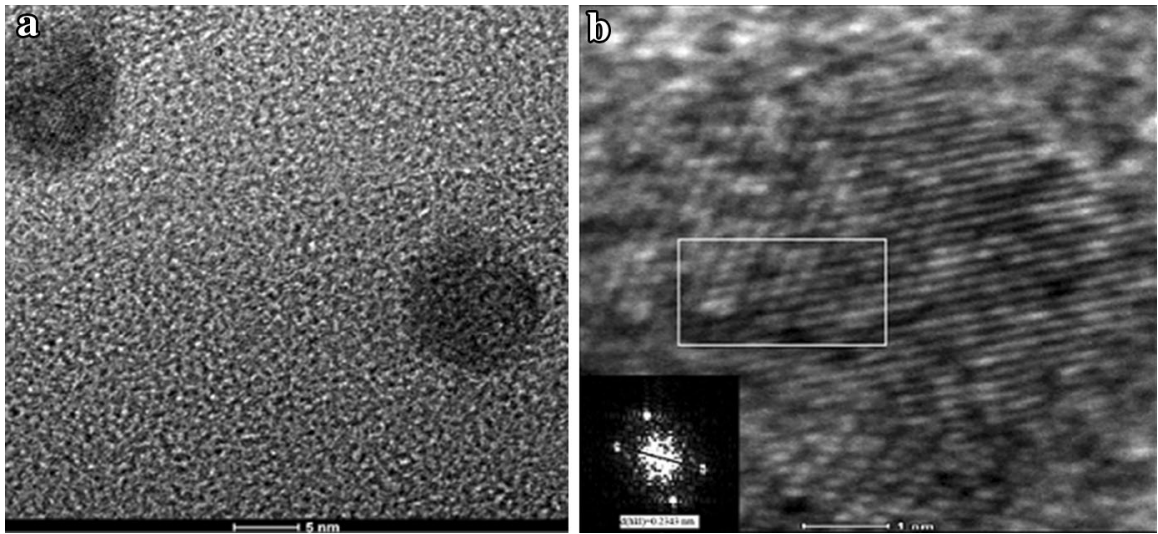

Fig. 9 HR-TEM images of PAA/Ag/MPA film: Ag nanoparticle at different magnification (a, b); marked square at Fig. 8b indicates the area selected for calculation of $d$ spacing, shown at left corner

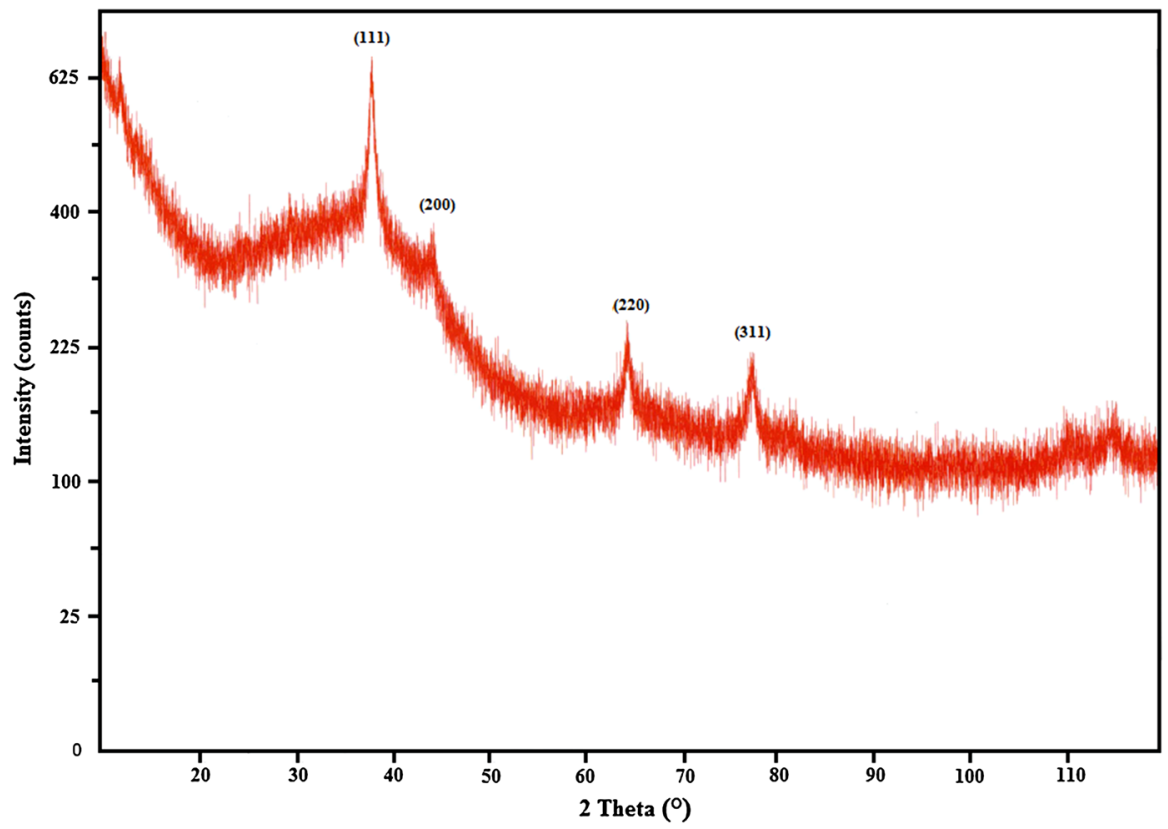

Fig. $10 \mathrm{XRD}$ pattern of PAA/Ag/MSA (peak indices at $2 \Theta$ positions)

(220), (311) planes of silver, are observed in both studied nanocomposites PAA/Ag/ MPA and PAA/Ag/MSA. These peaks are covered by a broad signal attributed to amorphous phase of polymer. Above results correspond well to data obtained for AgNPs presented by other authors [2, 12, 23]. 


\section{Atomic force microscopy (AFM)—adhesion and hardness measurements}

AFM has been used for measurement of nanomechanical properties of studied nanocomposites. Applying contact mode, the deflection-extension curves were recorded and then converted into force-extension curves (called also force-distance curves). This relation allows for designation of adhesion between nanocomposite sample and tip material (which was diamond) due to hysteresis, which is related to energy difference between loading and unloading process [28-30].

Adhesion between the sample and the tip is equivalent to the pull-off force during scanning process. The adhesion force $\left(F_{\mathrm{a}}\right)$ of poly(acrylic acid) or PAA/AgNPs nanocomposite was determined using Eq. 1, where $k$ is a spring constant of the cantilever, and $\Delta$ is the height of the peak on the force-distance curve during the unloading process $[20,29]$.

$$
F_{\mathrm{a}}=k \cdot \Delta
$$

The average of at least 40 measurements of adhesive forces for all samples is presented in Table 3 . As can be seen the value of $F_{\mathrm{a}}$ nanocomposites increases three times as compared with that of the PAA (unexposed). UV irradiation causes increase of adhesion in all samples but the changes are highest in pure, unmodified PAA. The increase in the force of adhesion in nanocomposites, caused by UV radiation, is similar for both samples (i.e., PAA/Ag/MPA and PAA/Ag/MSA).

The second characteristic feature of the nanomaterial is hardness $\left(H_{\mathrm{N}}\right)$, which is defined as a resistance to indentation. This parameter is calculated from the simple relationship:

$$
H_{\mathrm{N}}=F_{\max } / A
$$

where $F_{\max }$ is the maximum load applied, $A=f\left(h_{\mathrm{c}}\right)$ is the contact area between the tip and surface of the sample, which is directly related to indentation depth. $F_{\max }$ is estimated from the force-distance curves.

Analysis of residual impression (deformed surface) after tip removal leads to conclusion that our samples exhibit real elastic-plastic deformation behavior. An example of deformation pattern is shown in Fig. 11. Because it is well known that UV irradiation has great influence on surface properties of materials, the experiment has been repeated after exposure. Hardness measurements were made possible only

Table 3 Adhesion force and nanohardness of PAA, PAA/Ag/MPA and PAA/Ag/MSA (before and after 14-min UV irradiation) calculated on the base of AFM measurements

\begin{tabular}{lrll}
\hline Sample & Time of irradiation $(\mathrm{min})$ & Force of adhesion $(\mathrm{nN})$ & Hardness $(\mathrm{GPa})$ \\
\hline PAA & 0 & $2.09 \pm 0.35$ & $2.67 \pm 0.36$ \\
PAA & 14 & $5.83 \pm 0.39$ & $4.32 \pm 0.60$ \\
PAA/Ag/MPA & 0 & $6.14 \pm 0.20$ & $6.60 \pm 0.70$ \\
PAA/Ag/MPA & 14 & $8.10 \pm 0.29$ & $3.86 \pm 0.55$ \\
PAA/Ag/MSA & 0 & $6.03 \pm 0.32$ & $6.33 \pm 0.63$ \\
PAA/Ag/MSA & 14 & $8.13 \pm 0.35$ & $4.18 \pm 0.49$ \\
\hline
\end{tabular}




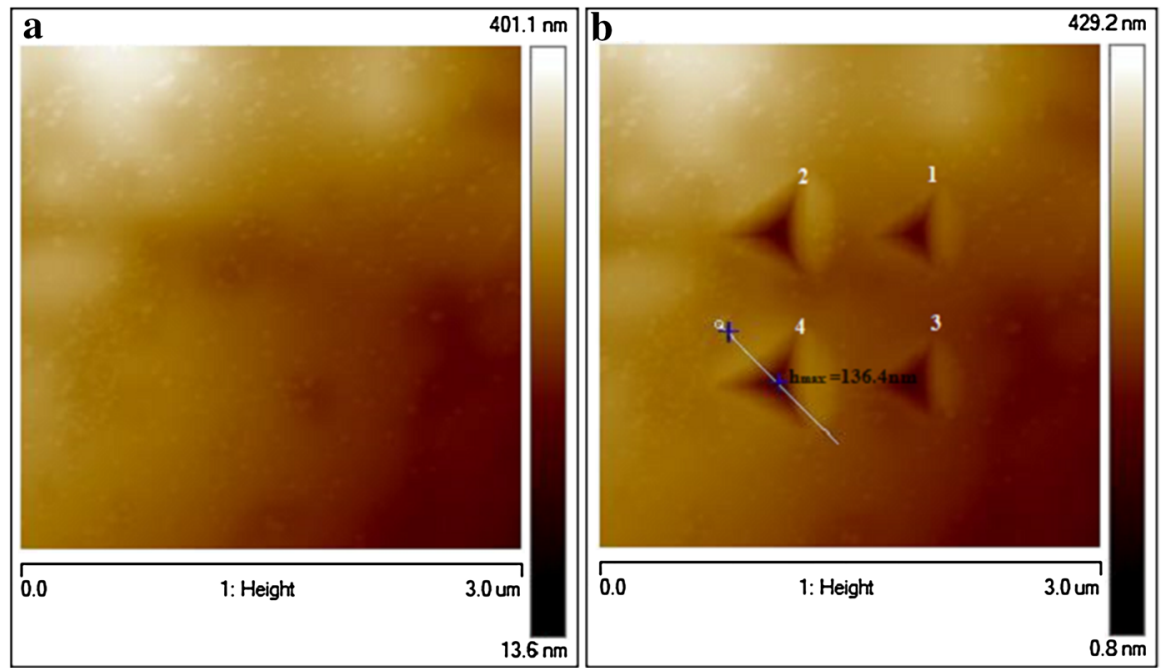

Fig. 11 Atomic force microscopy (height image) of PAA/Ag/MSA sample before (a) and after (b) the nanoindentation test

after a short time of UV irradiation, because prolonged irradiation caused photodegradation, resulting in crumbling samples.

The initial PAA nanohardness equals $2.67 \mathrm{GPa} \pm 0.36$ and increases above 1.6 times after 14-min exposure to UV. This increase can be explained by polymer crosslinking, which is a characteristic feature of PAA. The results indicate that photocrosslinking is very efficient at PAA surface. Similar behavior of PAA has been published by Nowicki et al. [31]. As stated in this work, both nanohardness and elastic indentation modulus in PAA increase by a factor about 1.5 after $8 \mathrm{~h}$ UV irradiation due to polymer photocrosslinking.

The opposite trend has been observed in UV-irradiated nanocomposites. Exposed samples PAA/Ag/MPA and PAA/Ag/MSA show an $\mathrm{H}_{\mathrm{N}}$ decline of 1.25 and 1.5 times, respectively. It can suggest that in the presence of Ag NPs, polymer photodegradation is dominant at the sample surface while crosslinking is inhibited. Greater effectiveness of photodegradation of PAA in nanocomposites it is probably due to the catalytic effect of nanosilver, suggested above.

\section{Summary and conclusions}

PAA was mixed with stabilized silver nanoparticles to obtain nanocomposites designed to biomedical and pharmaceutical applications.

Although the chemical structure of both used stabilizers is only slightly different (the difference in the number of carboxyl groups: 1 or 2; the presence of thiol group in the other position), MSA exhibits better stabilizing effect. Moreover, MSA is a more effective stabilizer in the nanocomposites exposed to UV light. 
In the presence of MPA, the formation of Ag nanoparticles is inhibited. Only the use of UV radiation, in addition to chemical reduction, allows for the creation of nanoparticles, which are characterized by typical plasmonic band in the spectrum of UV-Vis.

The formation of Ag nanoparticles at nanocomposite surface was confirmed by EDX analysis but the morphology of observed structures varies in the presence of MPA and MSA.

Long-term (12 months) storage of Ag/MPA solution does not cause significant changes in the absorption spectra (neither in the position nor in the intensity of the band). However, in the case of Ag/MSA, observed spectral changes indicate the slow agglomeration of NPs.

Thermogravimetric analysis allowed for estimation of stability upon heating and for determination of $\mathrm{Ag}$ content: $70 \%$ in stabilized NPs and $2 \%$ in nanocomposite.

PAA has the additional stabilizing effect on the nanoparticles; however, due to the susceptibility of PAA to the photodegradation, it does not cause a sufficient protection for nanocomposites with Ag NPs during prolonged irradiation.

Introduction of Ag NPs into PAA matrix caused the improvement of nanomechanical properties: both the adhesion of the samples to the tip and nanohardness increase relative to pure PAA. The differences related to the type of stabilizer are negligible.

However, UV irradiation has the opposite effect on the nanomechanical properties of pure PAA (dominant crosslinking) and nanocomposites (prevailing degradation).

The advantage of these systems from the point of view of biomedical applications is the presence of carboxylic groups that are capable of reacting with other functional moieties, e.g., amino groups present in proteins. It may be a potential material for the immobilization of enzymes or other catalysts, moreover, it can play an important role in removing impurities by physical adsorption.

Another important feature of the PAA is its ability to crosslink and form threedimensional network. In addition, the strongly hydrophilic poly(acrylic acid) has a unique capacity for the sorption of water, which is used in the production of hydrogels. They are used as dressings and hygienic materials, drug carriers, matrix for cell proliferation in tissue engineering, smart membranes sensitive to $\mathrm{pH}$, chemical sensors and many others.

The presence of Ag NPs in PAA, owing to theirs antimicrobial activity, gives the bactericidal properties of such nanocomposites, which ensures long-term use of these materials without exposure to infections.

Moreover, PAA as polyelectrolyte, similar to other conductive polymers [3], can be also used for production of medical sensors.

This is also important that the amount of silver in nanocomposites is small, and therefore it is expected that their production will be cheap.

Open Access This article is distributed under the terms of the Creative Commons Attribution 4.0 International License (http://creativecommons.org/licenses/by/4.0/), which permits unrestricted use, distribution, and reproduction in any medium, provided you give appropriate credit to the original author(s) and the source, provide a link to the Creative Commons license, and indicate if changes were made. 


\section{References}

1. Folarin OM, Sadiku ER, Maity A (2011) Polymer-noble metal nanocomposites: review. Int J Phys Sci 6:4869-4882

2. Sumesh J, Bootharaju MS, Pradeep AT (2011) A practical silver nanoparticle-based adsorbent for the removal of $\mathrm{Hg}^{2+}$ from water. J Hazard Mater 189:450-457. doi:10.1016/j.jhazmat.2011.02.061

3. Sargent A, Loi T, Gal S, Sadik OA (1999) The electrochemistry of antibody-modified conducting polymer electrodes. J Electroanal Chem 470:144-156. doi:10.1016/S0022-0728(99)00231-4

4. Zhang Y, Zhang K, Ma H (2009) Electrochemical DNA biosensor based on silver nanoparticles/ poly(3-(3-pyridyl) acrylic acid)/carbon nanotubes modified electrode. Anal Biochem 387:13-19. doi:10.1016/j.ab.2008.10.043

5. Wei Q, Li B, Li C, Wang J, Wang W, Yang X (2006) PVP-capped silver nanoparticles as catalysts for polymerization of alkylsilanes to siloxane composite microspheres. $\mathrm{J}$ Mater Chem 16:3606-36085. doi:10.1039/b608643h

6. Sinel'nikov BM, Kargin NI, Khoroshilova SE, Yasnaya MA, Kornilov DY, Sytnikov EV (2008) Silver-polystyrene composite material for electrochemical analysis. Pat RU 2(339):027

7. Fateixa S, Gira AV, Nogueira HIS, Trindade T (2011) Polymer based silver nanocomposites as versatile solid film and aqueous emulsion SERS substrates. J Mater Chem 21:15629-15636. doi:10. 1039/C1JM12444G

8. Chaloupka K, Malam Y, Seifalian AM (2010) Nanosilver as a new generation of nanoproduct in biomedical applications. Trends Biotech 28:580-588. doi:10.1016/j.tibtech.2010.07.006

9. He S, Yao J, Jiang P, Shi D, Zhang H, Xie S, Pang S, Gao H (2001) Formation of silver nanoparticles and self-assembled two-dimensional ordered superlattice. Langmuir 17:1571-1575. doi:10.1021/ $1 \mathrm{a} 001239 \mathrm{w}$

10. Li K, Fu-Shen E (2010) A novel approach for preparing silver nanoparticles under electron beam irradiation. J Nanopart Res 12:1423-1428. doi:10.1007/s11051-009-9690-2

11. Manz C, Williams L, Mohseni R, Zlotnikov E, Vasiliev A (2011) Dispersibility of organically coated silver nanoparticles in organic media. Coll Surf A Physicochem Eng Aspects 385:201-205. doi:10. 1016/j.colsurfa.2011.06.009

12. Zielinska A, Skwarek E, Zaleska A, Gazdac M, Hupka J (2009) Preparation of silver nanoparticles with controlled particle size. Procedia Chem 1:1560-1566. doi:10.1016/j.proche.2009.11.004

13. Amjad Z (2002) Water soluble polymers. Kluwer Academic Publishers, New York

14. Kaczmarek H, Szalla A (2006) Photochemical transformation in poly(acrylic acid)/poly(ethylene oxide) complexes. J Photochem Photobiol A:Chem 180:46-53. doi:10.1016/j.jphotochem.2005.09. 014

15. Li H, Liu B, Xi Zhang, Gao C, Shen J, Zou G (1999) Single-molecule force spectroscopy on poly(acrylic acid) by AFM. Langmiur 15:2120-2124. doi:10.1021/1a9800304

16. Zhang J, Oyama M (2007) Electrocatalytic activity of three-dimensional monolayer of 3-mercaptopropionic acid assembled on gold nanoparticle arrays. Electrochem Comm 9:459-464. doi:10. 1016/j.elecom.2006.10.015

17. Castro JL, López-Ramirez MR, Arenas JF, Otero JC (2004) Surface-enhanced Raman scattering of 3-mercaptopropionic acid adsorbed on a colloidal silver surface. J Raman Spectrosc 35:997-1000. doi: $10.1002 /$ jrs. 1247

18. Ścigalski F, Kaczmarek H, Wolnicka M (2013) Preparation and properties of silver nanoparticles stabilized by mercaptosuccinic acid with a controllable size. Adv Sci, Eng and Med 5:219-223. doi:10.1166/asem.2012.1256

19. Kaczmarek H, Metzler M, Ścigalski F (2014) Photochemical stability of poly(acrylic acid)/silver nanocomposites. Mater Lett 135:110-114. doi:10.1016/j.matlet.2014.07.161

20. Kaczmarek H, Gałka P (2011) Nano-mechanical properties of modified poly(methyl methacrylate) films studied by atomic force microscopy. Tribol Lett 41:541-554. doi:10.1007/s11249-010-9732-z

21. Gałka P (2011) Effect of UV-irradiation on selected properties of poly(methyl methacrylate) thin solid films modified by organic photosensitive compounds. PhD Dissertation. Nicolaus Copernicus University in Toruń

22. Sionkowski G, Kaczmarek H (2010) Polymers with silver nanoparticles-selected system-preparation, properties and uses. Polimery 55:545-551

23. Janardhanan R, Karuppaiah M, Hebalkar N, Rao TN (2009) Synthesis and surface chemistry of nano silver particles. Polyhedron 28:2522-2530. doi:10.1016/j.poly.2009.05.038 
24. Kaczmarek H, Metzler M (2014) The properties of poly(acrylic acid) modified with $N$-phenylbenzothioamide as potential drug carriers. Open Process Chem J 6:1-7. doi:10.2174/ 1875180601406010001

25. Dubinsky S, Graderb GS, Shterb GE, Silverstein MS (2004) Thermal degradation of poly(acrylic acid) containing copper nitrate. Polym Degrad Stab 86:171-178. doi:10.1016/j.polymdegradstab. 2004.04.009

26. Lepine L, Gilbert R (2002) Thermal degradation of polyacrylic acid in dilute aqueous solution. Polym Degrad Stab 75:337-345. doi:10.1016/S0141-3910(01)00236-1

27. Satapathy S, Shukla SP, Sandeep KP, Singh AR, Sharma N (2015) Evaluation of the performance of an algal bioreactor for silver nanoparticle production. J Appl Phycol 27:285-291. doi:10.1007/ s10811-014-0311-9

28. Balabanava N, Rymuza Z, Chizhik SA (2006) Estimation of Young's modulus and adhesive force of polymeric films by use of atomic force microscope. Metrol Meas Syst 13:253-260

29. Oliver WC, Pharr GM (1992) An improved technique for determining hardness and elastic modulus using load and displacement sensing indentation experiments. J Mater Res 7:1564-1583

30. Kurland NE, Drira Z, Yadavalli VK (2012) Measurement of nanomechanical properties of biomolecules using atomic force microscopy. Micron 43:116-128. doi:10.1016/j.micron.2011.07.017

31. Nowicki M, Richter A, Wolf B, Kaczmarek H (2003) Nanoscale mechanical properties of polymers irradiated by UV. Polymer 44:6599-6606. doi:10.1016/S0032-3861(03)00729-8 